\title{
A MULTI-PATH ALGORITHMIC APPROACH TO PHASED ARRAY CALIBRATION
}

\author{
Justine Mc Cormack, Tim Cooper and Ronan Farrell \\ Centre of Telecommunications Value Chain Research, \\ Institute of Microelectronics and Wireless Systems, \\ National University of Ireland Maynooth, \\ Co. Kildare, Ireland. \\ jmccormack@eeng.nuim.ie, tcooper@eeng.nuim.ie,rfarrell@eeng.nuim.ie
}

Keywords: Phase Array Antennas, Calibration, Tower-Top, Distributed Source Array, Autocalibration.

\begin{abstract}
The performance of phased arrays is dependent upon the amplitude and phase relationships between the elements of the array. In the presence of finite manufacturing tolerances and environmental effects these relationships cannot always be guaranteed, therefore synchronisation of these relationships is necessary. This paper presents an algorithmic approach to the calibration of these relationships, backed up with simulation results and comparisons.
\end{abstract}

\section{Introduction}

Adaptive antenna systems have been used for several years $[10-11,17]$. Their performance is dependent upon the amplitude and phase relationships between elements [27], which are affected by multiple environmental effects and by manufacturing variations in system components. These effects cause imbalances in the amplitude and phase relationships from such potential causes as: thermal effects, antenna mutual coupling, component aging and finite manufacturing tolerances [1, 9, 21 - 23]. There are several different approaches taken to solving this synchronisation problem, which range from fixed feeder paths $[2,8,14-15$, $18,25]$ to calibration algorithms [4 - 5, $19-22,24,26$, ]. Calibration algorithms are generally used in conjunction with fixed feeder paths, as the transceiver electronics located at the tower bottom also requires synchronisation. In situations where the transceiver electronics are connected directly to the antenna elements, synchronisation may only be achieved through the use of calibration algorithms. An example of such a setup is the tower top deployment of basestation electronics presented in [4 - 5].

This paper presents a calibration algorithm for a tower top system. The basestation electronics are deployed to the tower top, where each element of the array is connected to a transceiver element. The calibration algorithm uses additional distributed measurement elements and takes advantage of the structure of the array to minimise the complexity of the calibration challenge.

The paper is laid out as followed: section 2 presents an introduction to the tower top system. Which is followed by a description of the calibration algorithm in section 3 . Finally an evaluation of the performance of the calibration algorithm is presented in section 4 .

\section{The Tower Top Antenna Array}

A tower top system has the basestation electronics redeployed to the tower top, where each element of the array has its own transceiver element. These arrays are generally planar arrays from 16 to 64 elements, as planar arrays are more compact than circular arrays $[6,12]$. Tower top array synchronisation is generally done by either radiative or non-radiative calibration.

The system presented here uses non-radiative calibration to remove the need for external calibration equipment. The calibration algorithm uses the structure of the array to simplify the calibration of large arrays. The array is a planar array interlaced with reference elements, as shown in figure 1. Each reference element is connected to four transceiver elements via directional couplers [3]. This interconnection structure provides at least one calibration path for each of the transceiver elements of the array. These calibration paths, as shown in figure 2 , are completed by digital feedback from the reference element to each of the transceiver elements.

The planar array structure of the system can be considered in terms of building blocks. The array consists of reference elements; each reference element is surrounded by four transceiver elements. If you consider one reference element surrounded by the four transceiver elements as a single tile, then the whole array can be considered just a construct of overlapping tiles. The reason for considering the array in this way is that a single reference element surrounded by four elements is basically a circular array. The layout looks square but each of the antennas is equidistant from the reference element, thus describing a circle. The advantage of using small circular arrays and tiling them to produce a larger array is that the scaling problem of circular arrays is overcome. Circular arrays are difficult to scale for a number of reasons $[6,12]$ for example, the larger the array the more area the array requires [16], fixed feeder paths are required to connect each element to the central reference element which can require long looped cables, and there is also a physical limitation to the number of connections a single reference element can handle. Thereby tiling small circular arrays together a scalable array can be constructed with a scalable calibration mechanism. 


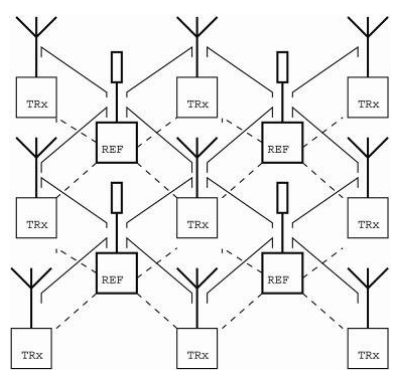

Figure 1: Tower-Top, Cellular, Phased Array Antenna System.

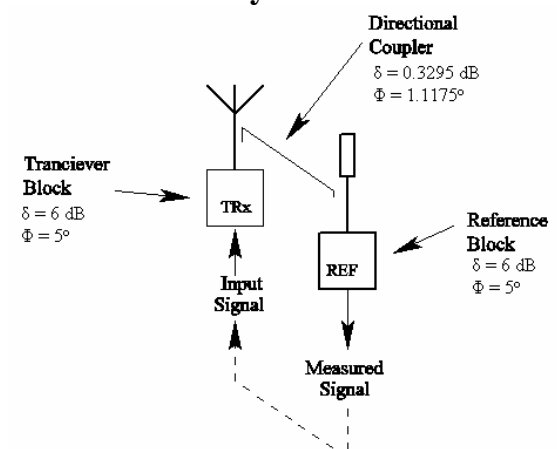

Figure 2: The Calibration Path of an Antenna Element.

\section{Algorithmic Approaches}

The algorithmic approach presented in this paper is compared with the shortest path algorithm, the best performing algorithm presented to date for this structure [4]. Both algorithms are based upon comparisons between elements, so a brief description of the shortest path algorithm is presented first, followed by a description of the new algorithm and a comparison of the simulation results of both algorithms.

\subsection{Shortest Path Calibration Algorithmic Approach}

The shortest path algorithm is based upon comparisons between elements. These comparisons start out from a reference transceiver element in the array; this element is measured by a reference element connected to it. Then another element connected directly to the same reference element is measured. The measured signals are then compared; the correction factor from this comparison is feed back into the second element to calibrate it to the reference transceiver element. These comparisons are continued throughout the array, by using intermediate reference transceiver elements and by calibrating around the reference elements in a similar way. This has the effect of removing the imbalances in the amplitude and phase relationships of the array due to the reference blocks, as each of the comparisons use measurements taken from the same reference elements. The reference element variations for the two compared measurements are the same so the reference element variations do not affect the correction factors. The comparisons also have the effect of removing individual transceiver block variations, as the comparisons of the transceiver elements are corrected to the reference transceiver' specific imbalance.
The elimination of the component block variations is only possible if the measurement taken by the reference blocks is accurate. This accuracy is affected by not only the measurement but also by the resolution of the analog to digital converters (ADC), because of the digital feedback of the system. These challenges can be over come by using high resolution $\mathrm{ADCs}$ in conjunction with a measurement technique such as cordic [19], or by themselves. However these issues will not be discussed in detail as they are beyond the scope of this paper.

Due to the elimination of the component block variations, the overall array variation is dependent on the number of coupler variations that affect each element of the array, due to its correction factor. The correction factor generated from each comparison includes a coupler variation. This coupler variation is a composite of the coupler variations included in each of the measured signals in the comparison. As the number of comparisons required to calibrate the whole array increases, so does the number of coupler variations included in the correction factors, therefore the longer the calibration trail is to an element, the more coupler errors included in its correction factor. So each element's accuracy is dependent on its correction factor, the further away it is from the reference transceiver element, the greater the number of coupler variations that affect it, and the less accurate it is.

The accuracy of the array is dependent upon the number of couplers along the calibration trials from the reference transceiver elements to rest of the array. The accuracy can be improved by shortening the length of these paths, so by moving the reference transceiver element to the center of the array, the maximum distance from the reference transceiver element is shortened. By only calibrating previously uncalibrated elements the accuracy of the calibration is also improved, as calibrated elements are not recalibrated with less accurate correction factors due to longer paths.

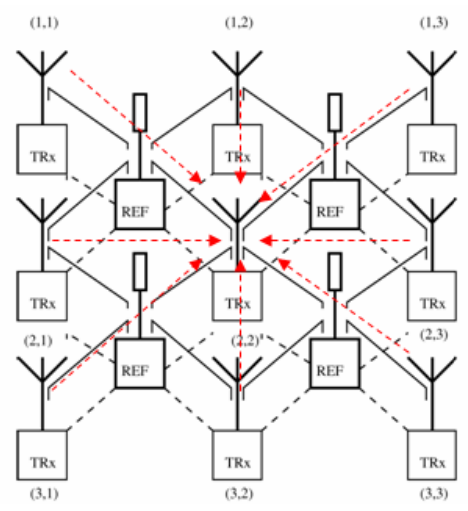

Figure 3: Comparison of the shortest path algorithm on a $3 \times 3$ array.

The shortest path algorithm calibrates the shortest path to each element, as shown in figure 3 , for a three by three array. The shortest path algorithm calibrates in rings around the reference transceiver element, calibrating the elements directly connected to the reference transceiver element to it. Then as it moves further out, the next ring of elements are calibrated to the ring of previously calibrated elements directly connected to them, and so forth until the entire array is calibrated. This has the advantage of calibrating each 
element along the shortest route from the reference transceiver element to it, and only calibrating each element once. This type of comparison algorithm maximises the performance of a single step comparison approach.

The accuracy of this algorithm can be predicted by calculating the number of couplers which affect each element of the array. The following expressions present in terms of odd and even $n, n^{2}=N, N$ is the number of elements in the array.

Even $n$ :

$$
\sigma_{a k}^{2}=\left(\frac{\sigma_{c k}^{2}}{N-1} \sum_{i=1}^{\frac{n}{2}-1} 8 i^{2}\right)+\left(\frac{2 n-1}{N-1}\right)\left(\frac{n}{2}\right) \sigma_{c k}^{2}
$$

Odd $n$ :

$$
\sigma_{a k}^{2}=\left(\frac{\sigma_{c k}^{2}}{N-1} \sum_{i=1}^{\frac{n-1}{2}} 8 i^{2}\right)
$$

Where, $\sigma_{a k}^{2}$ and $\sigma_{c k}^{2}$ are the RMS array variance and the RMS coupler variance respectively.

\subsection{Dual Path Calibration Algorithmic Approach}

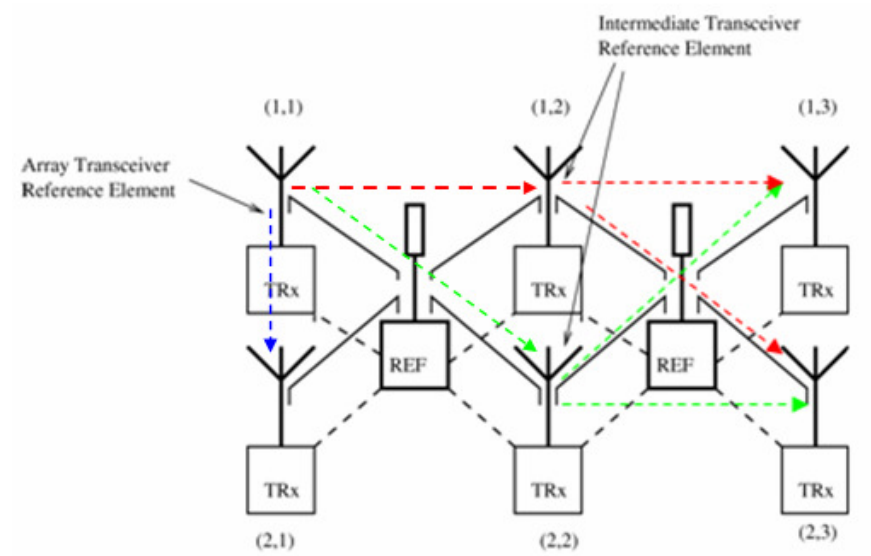

Figure 4: Dual Path Calibration Comparisons for a 2x3 Array.

This proposed algorithm differs from other comparison based algorithms as it takes two routes to elements, where available, in order to reduce the effect of the coupler variation. This is achieved by taking two routes of the same length to any element, as shown in figure 4. The elements directly connected to the reference transceiver element are calibrated directly to the reference transceiver element, the same way as the shortest path algorithm. The elements further away are calibrated using two paths of the same length, each of these paths generate a correction factor for the element they are calibrating. These correction factors are averaged; thus statistically reducing the effect of couplers along the routes. This averaging reduces the effect of outlier coupler elements, but can also increase the variation of couplers with very small variations.

As the accuracy of the algorithm is affected by the coupler variations, the algorithm's accuracy can be predicted by calculating the number of coupler variations that affect each element of the array. The following expressions calculate the accuracy of the array relative to the reference transceiver element, which is not affected by coupler variations. The representation of the array in this way is conveniently expressed in terms of odd and even $n$, where $n^{2}=N, N$ is the number of elements in the array.

Even $n$ :

$$
\sigma_{a k}^{2}=\left(\frac{8 \sigma_{c k}^{2}}{N-1}\right)+\left(\frac{\bar{\sigma}_{c k}^{2}}{N-1} \sum_{i=2}^{\frac{n}{2}-1} 8 i^{2}\right)+\left(\frac{2 n-1}{N-1}\right)\left(\frac{n}{2}\right) \bar{\sigma}_{c k}^{2}
$$

Odd $n$ :

$$
\sigma_{a k}^{2}=\left(\frac{8 \sigma_{c k}^{2}}{N-1}\right)+\left(\frac{\bar{\sigma}_{c k}^{2}}{N-1} \sum_{i=2}^{\frac{n-1}{2}} 8 i^{2}\right)
$$

Where $\sigma_{a k}^{2}$ and $\sigma_{c k}^{2}$ are the RMS array variance and the RMS coupler variance respectively and $\bar{\sigma}_{c k}^{2}$ is the averaged RMS coupler variance. The first term in each expression represent the eight elements directly connected to the reference transceiver element, and are calibrated by only one path each. For small array sizes less than a $4 \times 4$ array, the performance of the single-path algorithm $(1,2)$ and that of the dual-path approach $(3,4)$ are the same, as all elements are directly connected to the reference transceiver and thus can only be calibrated along one path. As the size of the array increases then the number of coupler variations increase, this is a straight forward calculation for the shortest path algorithm prediction equations (1) and (2). However, for the dual path algorithm prediction equations (3) and (4) the calculation is slightly different. The first term in equation (3) and (4) is a calculation of the eight elements of the array directly surrounding the central reference transceiver element; which is calibrated by a single path. The remaining elements of the array are calibrated by dual paths. From (3) and (4) the second and third terms calculate the number of elements calibrated by averaged coupler variations.

\section{Simulation Results}

Both algorithms have been simulated using Matlab, and have been implemented on a Matlab model, where each of the antenna chains is composed of component blocks of the system, as shown in figure 2, which are given a random variation in line with the manufacturing tolerances of that particular component block, as shown in table 1. Each transceiver is feed with a $20 \mathrm{~dB}$ signal that varies with a 0.5 $\mathrm{dB}$ standard deviation, and a random phase. These are the base components of the models upon which simulations of the algorithms were performed.

\begin{tabular}{|c|c|c||c|c|}
\hline Component $(\mathrm{i}, \mathrm{j})$ & $\mu_{(\mathrm{i}, \mathrm{j}) \mathrm{A}}$ & $\Sigma_{(\mathrm{i}, \mathrm{j}) \mathrm{A}}$ & $\mu_{(\mathrm{i}, \mathrm{j}) \Phi}$ & $\Sigma_{(\mathrm{i}, \mathrm{j}) \Phi}$ \\
\hline Tx S21 & $50 \mathrm{~dB}$ & $3 \mathrm{~dB}$ & $10^{\circ}$ & $5^{\circ}$ \\
\hline Ref S21 & $60 \mathrm{~dB}$ & $6 \mathrm{~dB}$ & $85^{\circ}$ & $5^{\circ}$ \\
\hline Coupler S21 & $-20.3295 \mathrm{~dB}$ & $0.3295 \mathrm{~dB}$ & $90.197^{\circ}$ & $1.1175^{\circ}$ \\
\hline
\end{tabular}

Table 1: Component Block Imbalances. 


\subsection{Comparison of Theory and Simulation}

Ten thousand simulations of the dual path algorithm were run to give a statistically significant result for square arrays ranging in size from a $2 \times 2$ array to a $10 \times 10$ array. These simulations were compared to the predicted accuracy of the dual path algorithm as estimated by (3) and (4). This comparison is shown in figure 5. Due to the prediction equations being based upon standard deviations of the couplers, the averaged coupler terms are estimated by a percentage reduction in the coupler standard deviation. The percentage reduction is calculated based upon size of the array, and therefore scales up as the size of the array increases. As can be seen in figure 5, this is a good approximation of the accuracy of the algorithm. The RMS array error increases as the size of the array increases due to the dependency of the array accuracy on coupler variations.
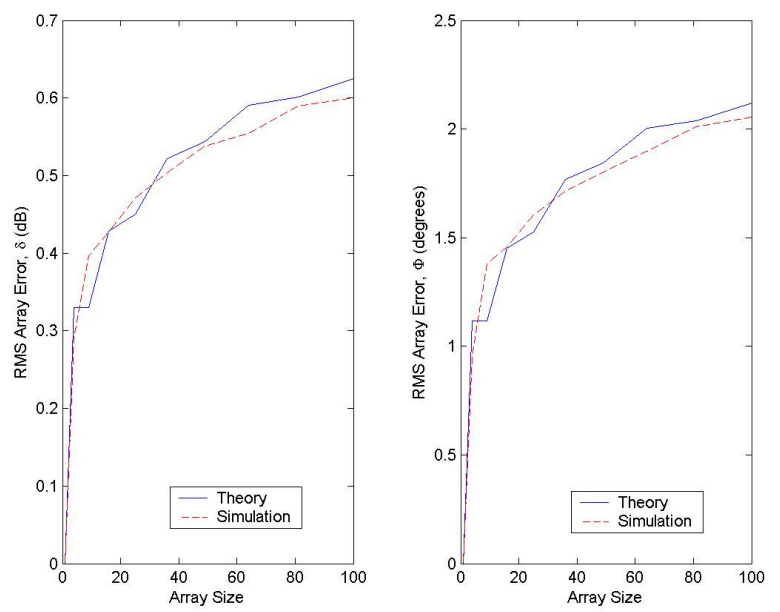

Figure 5: The overall array calibration accuracy predicted by equation 1 and 2 and calibration simulations.

\subsection{Comparison of the Two Calibration Algorithms Simulation Results}

A comparison between the two algorithms, shortest path algorithm and dual path algorithm, is presented in figure 6 . Again 10,000 simulations of each size array are taken as a statistically significant measure. These results show that as the size of the array increases so does the RMS array error, as the calibration routes increase for each element, the more coupler variations that are included. Figure 6 clearly shows that as the size of the array increases so does the dual path algorithm performs improve in comparison to the shortest path algorithm performance. This is due to both algorithms performance being dependent upon the number of coupler variations included in each element's correction factors. The dual path algorithm however uses an averaging of two paths of identical lengths to reduce the effect of the coupler variations, which will have more of an effect on the output of larger arrays as dual path calibration will be performed on proportionally more elements.
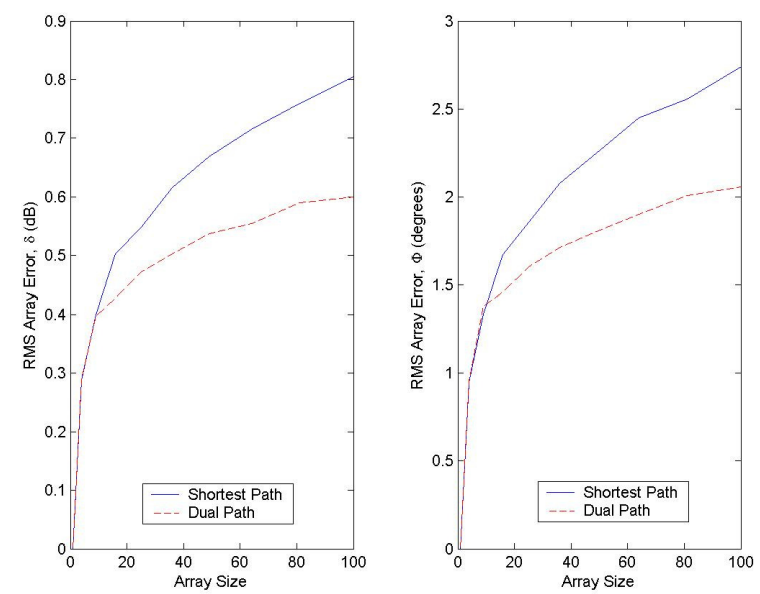

Figure 6: Comparison of RMS Array Error of Shortest Path and Dual Path Calibration Algorithms, as the Size of the Array increases.

A comparison of the number of elements that fall within the absolute array variations is shown in figures 7 and 8 . This is based upon 10,000 simulations of a five by five array. The results are consistent with the previous set of comparison results, as the first $30 \%$ of elements (approximately 8 elements per array) are exactly the same as that of the shortest path. Which is consistent with the two algorithms, as they both share a ring of elements that surround the reference transceiver element that are calibrated in exactly the same way. The results diverge at this point, which is consistent with the dual path averaging of the correction paths to reduce the overall error.

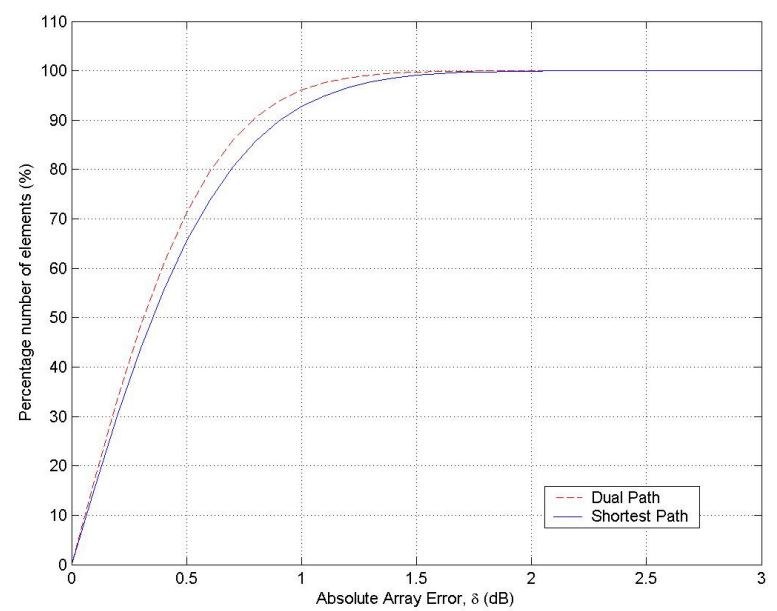

Figure 7: Percentage Number of Elements vs. the Absolute Amplitude Variation. 


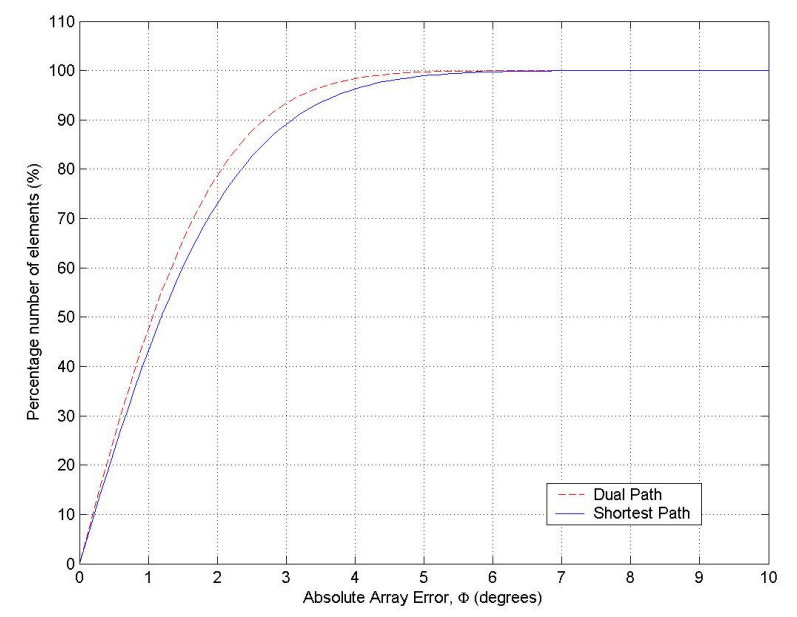

Figure 8: Percentage Number of Elements vs. the Absolute Phase Variation

\section{Conclusions}

Synchronisation of phased arrays is of vital importance to the performance of the array. This synchronisation can be achieved through a combination of, or solely by, fixed feeder networks and calibration algorithms. This paper focuses on novel calibration algorithms that utilises the tessellated structure of rectilinear arrays. A scalable method was presented for calibrating planar arrays using a built-in nonradiative calibration mechanism. The structure of the array provides multiple calibration paths for the elements of the array. The dual path algorithm utilises these multiple paths to improve the accuracy of the array. The dual path algorithm presented simulation results that show improvement over the previously presented shortest path calibration algorithm for this system. It shows a marked improvement as the array size increases, to an improvement of up to $0.2047 \mathrm{~dB}$ and $0.6833^{\circ}$. This improvement is down to the reduction of the directional coupler variation effect on the RMS array error variation, by averaging two paths of the same length to achieve this reduction. Future work will focus on the implementation of a $4 \times 4$ array prototype.

\section{Acknowledgements}

Special thanks to Science Foundation Ireland (SFI) for their generous funding of this project through the Centre of Telecommunications Value Chain Research (CTVR).

\section{References}

[8] M. Adibi, K. Clements, R. Kafka and J. Stoval. "Remote Measurement Calibration", IEEE Computer Applications in Power, Vol. 3, Iss. 4, Oct. 1994, pp. 37 42.

[12] D. C. Chang, S. H. Jou, "The study of Butler Matrix BFN for 4 Beams Antennas System", IEEE International Symposium on Antennas and Propagation, Vol. 4, June 2003, pp. 176 - 179.

[23] T. S. Cooper, G. Baldwin and R. Farrell "Six - port
Precession Directional Coupler", Electronic Letters, Vol. 42, No. 21, 12 Oct. 2006, pp. 1232 - 1234.

[17] T. S. Cooper, J. Mc Cormack, R. Farrell and G. Baldwin. "Towards Scalable, Automated Tower - Top Phased Array Calibration", in Vehicular Technology Conference, Dublin Ireland April 23 - 25, 2007.

[16] T. S. Cooper, J. Mc Cormack, and R. Farrell. "Tower Top Antenna Array Calibration Scheme for Next Generation Networks", Eurosip 2007, accepted.

[25] E. D. Di Claudio, "Asymptotically Perfect Wideband Focusing of Multi-ring Circular Array", IEEE Trans. On Signal Processing, Vol. 53, No. 10, Oct. 2005, pp. 3661 $-3673$.

[22] L.C. Godara "Smart Antennas", CRC Press LLC, 2004, pp. 325 - 348.

[11] H. Hayashi, D. A. Hitko, C. G. Sodini, "Four - Element Planar Butler Matrix using half - wavelength open stubs", IEEE microwave and wireless components letters, Vol. 12, No. 3, March 2002.

[7] I. Jonsdottir and A. Hanksdottir. "Integrity Monitoring and Estimation of Systematic Errors in Radar Data Systems", IEEE Int. Radar Conference, May 1995, pp. $310-316$.

[2] T. Kaiser, et el. "When will smart antennas be ready for market? Part 1", IEEE Signal Processing Magazine, pp. 87 - 92, March (2005).

[3] T. Kaiser, et el. "When will smart antennas be ready for market? Part 2 - Results", IEEE Signal Processing Magazine, pp. 174 - 176, November (2005).

[24] M. Latman, "Circular Array STAP", IEEE Trans. On Aerospace and Electronic Systems, Vol. 36, No. 2, April 2000, pp. 510 - 517.

[21] R. J. Mailloux, "A Phased Array Error Correction Scheme", International Symposium on Antennas and Propagation Society, Vol. 1, June 1993, pp. 202 - 205.

[14] S. Mosca, F. Bilotti, A. Toscano and L. Vegni “A Novel Design Method for Blass Matrix Beamforming Networks", IEEE Trans. On Antennas and Propagation, Vol. 50, No. 2, Feb 2002.

[10] H. Nord, "Implementation of $8 \times 8$ - Butler Matrix in Microstrip", Diploma Thesis, Royal Institute of Technology Stockholm, Germany 2000.

[26] C. C. Phillips, "A New Approach to Antenna BeamShaping - The 'Coke - Bottle' Antennas", WESCON/60 Conference Record, Vol. 4, Part 1, Aug. 1960, pp. 74 82.

[1] F. Rayal. "Why have smart antennas not yet gained traction with wireless network operators?", IEEE Antennas and Propagation Magazine, Vol 47, No. 6, pp. 124 - 126, Dec (2005).

[13] K. Shetty, "A Novel Algorithm for Uplink Interference Suppression using Smart Antennas in Mobile Communications", Masters of Science Thesis, Florida State University 2004.

[27] C. Shipley and D. Woods, "Mutual Coupling - Based Calibration of Phased Array Antennas", IEEE International Conference on Phased Array Systems and Technology, 21 - 25 May 2000, pp. 529 - 532.

[20] K. Takao, M. Fujita and T. Nishi, "An Adaptive Antenna Array under Directional Constraint", IEEE Trans. On 
Antennas and Propagation, Vol. AP - 24, No. 5, Sept. 1976.

[5] G. Tsoulos, J. McGeehan, M. Beach. "Space Division Multiple Access (SDMA) Field Trials. Part 2: Calibration and Linearity Issues", IEE Proc. Radar, Sonar Navig. Vol. 145, No. 1, pp., February (1998).

[6] N. Tyler, B. Allen and H. Aghvami. "Adaptive Antennas: The Calibration Problem", IEEE Comms. Magazine, Vol. 42, Iss. 12, Dec. 2004, pp. 114 - 122.

[9] N. Tyler, B. Allen, A. H. Aghvami. "Calibration of Smart Antenna Systems: Measurements and Results", IET Microwaves, Antennas and Propagation, Vol. 7, Iss. 3, June 2007, pp. 629 - 638.

[19] B. O. Van Veen and K. M. Buckley, "Beamforming: A Versatile Approach to Spatial Filtering", IEEE ASSP Magazine, Vol. 5, Iss. 2, April 1988, pp. 4 - 24.

[15] S. L. Vetterlein, P. S. Hall, "Multiple Beam Microstrip Patch Array with Integrated Beamformer", IEE Proceedings, Vol. 138, No. 2, April 1991.

[18] J. E. Volder, "The CORDIC Trigonometric Computing Technique", IRE Trans. Comp., Vol. EC-8, No. 3, 1959, pp. 330 - 334.

[4] H. S. C. Wang, "Performing of Phased Array Antennas under Error Conditions", IEEE Aerospace applications Conference Digest. 12 - 17 Feb. 1989, pp. 1 - 25. 